Dear Editor,

Please find enclosed the manuscript titled "Evaluation of Helicobacter pylori eradication by triple therapy plus Lactobacillus acidophilus compared to triple therapy alone" by Medeiros JAS et al. for consideration for publication in the European Journal of Clinical Microbiology \& Infectious Diseases.

The named authors have seen and agreed to the submitted version of the paper. The content is original and has not been published elsewhere, and the manuscript is not currently in submission for publication elsewhere.

If the contribution is accepted for publication, the paper will not be published elsewhere in the same form, in English or in any other language, without written consent of the copyright holder.

We are looking forward to receiving your answer.

With kind regards,

Jose Augusto da Silva Medeiros, MD, PhD 


\section{EVALUATION OF HELICOBACTER PYLORI ERADICATION BY TRIPLE THERAPY PLUS LACTOBACILLUS ACIDOPHILUS COMPARED TO TRIPLE THERAPY ALONE}

José Augusto da Silva Medeiros, MD, $\mathrm{PhD}^{1}$, Teresa Maria Fonseca Oliveira Gonçalves, MSc, $\mathrm{PhD}^{2}$, Lyudmila Boyanova MD, $\mathrm{PhD}^{3}$, Marta Isabel de Correia Pereira, $\mathrm{MD}^{1}$, José Nuno da Silva Paiva de Carvalho, $\mathrm{MSc}^{4}$, Anabela Maria de Sousa Pereira MSD, $\mathrm{PhD}^{5}$, António Manuel Silvério Cabrita, $\mathrm{MD}, \mathrm{PhD}^{4}$

${ }^{1}$ Institute of Physiology, Faculty of Medicine, University of Coimbra, Coimbra, Portugal, ${ }^{2}$ Institute of Microbiology, Faculty of Medicine, University of Coimbra, Coimbra, Portugal, ${ }^{3}$ Department of Microbiology, Medical University of Sofia, Sofia, Bulgaria, ${ }^{4}$ Institute of Pathology, Faculty of Medicine, University of Coimbra, Coimbra, Portugal, ${ }^{5}$ Department of Educational Sciences, University of Aveiro, Aveiro, Portugal

Corresponding author: Assoc. Prof. José Augusto da Silva Medeiros, Rua do Carmo, No. 54, 4V, 3000-098 Coimbra, Portugal. E-mail: jmedeiros@fmed.uc.pt; telephone: 919502495 


\begin{abstract}
Purpose To evaluate the influence of adding Lactobacillus acidophilus to a triple regimen for H. pylori eradication, in untreated patients with peptic ulcers or ulcer-scars. Methods: Prerandomized, single-blind, interventional, treatment-efficacy study with active controls and parallel-assignment, set in Coimbra, Portugal, on 62 consecutive H. pylori-positive untreated adults with peptic ulcers or ulcer-scars, diagnosed by gastroduodenoscopy, with pre-treatment direct Gram-staining and culture of gastric biopsies. The first 31 patients received esomeprazole $20 \mathrm{mg}$, amoxicillin 1000mg and clarithromycin 500mg (EAC), all b.i.d., for 8 days. The remaining 31 added L. acidophilus, $5 \times 10^{9}$ organisms per capsule, $3+2$ i.d. for 8 days (EACL). Main outcome measure: ${ }^{13} \mathrm{C}$ urea breath test (UBT), $\geq 6$ weeks after completion of therapy. Results: Successful eradication (UBT-negativity after treatment), was similar in both groups $(\mathrm{EAC}=80.6 \%$; $\mathrm{EACL}=83.9 \%, \mathrm{p}=0.740)$ by both intention-to-treat and perprotocol analysis. The non-eradicated strains were susceptible in vitro to both antibiotics. Conclusion: Adding L. acidophilus to EAC triple therapy did not increase H. pylori eradication rate. Considering the cost and the burden of ingesting five extra capsules daily, supplementing the EAC therapy with L. acidophilus, at this dose, shows no benefit. Further studies with different dosages and duration, and other probiotics or probiotic combinations are required to improve eradication.
\end{abstract}

Keywords: Helicobacter pylori, Lactobacillus acidophilus, triple therapy, eradication, urea breath test, clarithromycin, amoxicillin

The study was presented at the 15th United European Gastroenterology Week (UEGW), 2731 October 2007, Paris, France. Gut 2007; 56 (Suppl III) A101 


\section{INTRODUCTION}

H. pylori infection is associated with chronic gastritis and peptic ulcer disease, and is a risk factor for the development of gastric cancer and MALT lymphoma. ${ }^{1}$ Successful eradication of the infection by triple associations of antibiotics and proton-pump inhibitors is curative. However, the treatment may fail in $10-35 \%$ of all cases due to $H$. pylori resistance, antibiotic side effects and other reasons [1]. Searching for new or additional therapeutic agents is necessary to overcome treatment failures.

There is an ongoing controversy in the literature regarding the usefulness of the addition of probiotics, including Lactobacillus acidophilus, to the standard triple therapy for H. pylori eradication [2-7]. While the activity of L. acidophilus, L. johnsonii and L. casei subsp. rhamnosus strains against $H$. pylori has been demonstrated in vitro, the in vivo successful $H$. pylori eradication by lactobacilli has been uncommon $[2,8,9]$.

There are relatively few studies using triple regimens supplemented with $L$. acidophilus in humans $[5,6,7]$. The aim of the study was to evaluate the influence of adding $L$. acidophilus to a triple regimen for $H$. pylori eradication in untreated patients with peptic ulcers or ulcer scars.

\section{PATIENTS AND METHODS}

Patients. Eligibility criteria: previously untreated $H$. pylori-positive adults aged 20 years or over who had endoscopically confirmed peptic ulcers or peptic ulcer scars. Exclusion criteria: previous or current treatment with proton pump inhibitors or triple therapy, and neoplastic ulcers. Design. Sixty-two consecutive $H$. pylori-positive patients with peptic ulcers or peptic ulcer scars, undergoing upper gastrointestinal endoscopy in an outpatient clinic in Coimbra, Portugal, were enrolled in the study. Patient characteristics are summarized in Table 1. Before treatment of the $H$. pylori infection, eight gastric biopsy specimens were obtained per patient: two specimens from the body and two from the antrum were used for direct Gram staining, and the remaining two specimens from the body and two from the antrum were used for culture. The patients were considered as H. Pylori-positive only if the culture was positive $[10]$.

Culture and antibacterial susceptibility testing. The biopsies were frozen in Brucella broth with $25 \%$ glycerol and kept at $-80^{\circ} \mathrm{C}$ before being cultured, as previously described [11]. Immediately prior to culture, the biopsies were chopped and spread evenly onto H. pylori selective medium (PYL agar, Bio Mérieux, France). The plates were incubated for 3-10 days at $37^{\circ} \mathrm{C}$ under microaerophilic conditions (CampyGen system, Oxoid, UK). 
The suspected colonies were tested with a home-made rapid urease test (personal communication, L Monteiro, Lisboa, Portugal) and subcultured onto H. pylori selective medium (Wilkins-Chalgren agar with $10 \%$ horse blood, vancomycin $10 \mathrm{mg} / \mathrm{L}$, cefsulodin 5 $\mathrm{mg} / \mathrm{L}$, trimethoprim $5 \mathrm{mg} / \mathrm{L}$, and cycloheximide $100 \mathrm{mg} / \mathrm{L}$, Biogerm, Porto, Portugal). The plates were incubated at $37^{\circ} \mathrm{C}$ for $48 \mathrm{~h}$ in a microaerophilic atmosphere (CampyGen). $\mathrm{H}$. pylori identification was made by Gram staining and testing for the presence of oxidase, catalase, and urease activity.

The isolated $H$. pylori strains were subcultured. The minimal inhibitory concentrations (MICs) of clarithromycin and amoxicillin were evaluated against 31 strains from the patients in the EACL group and 28 strains from those in the EAC group. Three strains from the EAC group were lost while being subcultured. The strain susceptibility to the antibiotics used in this study was determined by $\mathrm{E}$ test, according to the method previously described $[11,12]$. The plates were incubated for $48 \mathrm{~h}$ in a microaerophilic atmosphere and were read according to the supplier's recommendations.

Intervention intended. A pre-randomization design was used [13]. To prevent a selection bias, the first 31 patients were allocated to therapy with esomeprazole $20 \mathrm{mg}$, amoxicillin $1000 \mathrm{mg}$ and clarithromycin $500 \mathrm{mg}$, all b.i.d. for eight days (EAC group). The remaining 31 patients were allocated to therapy with esomeprazole, amoxicillin and clarithromycin (as above) plus L. acidophilus, $5 \times 10^{9}$ bacteria per capsule, 3 capsules in the morning and 2 capsules at night, for the same period of time (EACL group), (Table 1). The $L$. acidophilus probiotic used was "Lacteol” (BioSaúde laboratories, Portugal). Given the nature of the study, the patients were not blinded to the assigned treatment.

Urea breath test. To detect possible differences in $H$. pylori eradication rates between the patients of EACL group and those of EAC group, a ${ }^{13} \mathrm{C}$ urea breath test (UBT, Helico-Test ${ }^{13}$ C-Ureia, Isomed S.L. Pharmaceutical Laboratory, Madrid, Spain) was performed six to seven weeks after the end of therapy. The specimen collection was performed immediately before (basal sample) and 30 min after (post-test sample) the ingestion of the marked urea; the ${ }^{13} \mathrm{C} /{ }^{12} \mathrm{C}$ ratio was measured in both specimens, and the result was expressed as the difference between the basal and post-test measurements. The UBT was considered positive when this value was $>5$ delta over baseline (DOB) per 1,000. This study had a single-blind design, with the technicians administering the UBT and evaluating the outcomes being blinded to patient group assignments.

Statistical analysis. The chi-square test and Fisher's exact test of independence (when appropriate) were used for the statistical analysis. The outcome variable was the presence of 
H. pylori eradication (yes vs no) and the exposure variable was Lactobacillus supplementation of the triple therapy (yes $\underline{v s}$ no). A $\mathrm{p}$ value of $<0.05$ was considered statistically significant.

This study was approved by the Ethics Committee of the Social Services of the University of Coimbra (Coimbra, Portugal) with the registration number 36/07. It was registered at ClinicalTrials.gov, of the National Institutes of Health (USA), with the protocol ID “SASUC-36/07”. Informed written consent was obtained from all patients.

\section{RESULTS}

The MICs of the 31 strains in the EACL group and the 28 strains in the EAC group for amoxicillin were $\mathrm{MIC}_{50},<0.016$ and $<0.016 \mathrm{mg} / \mathrm{L}, \mathrm{MIC}_{90}, 0.023$ and $0.023 \mathrm{mg} / \mathrm{L}$, and ranges of $<0.016-0.047$ and $<0.016-0.19 \mathrm{mg} / \mathrm{L}$, respectively. The MICs of the 31 strains in the EACL group and the 28 strains in the $\mathrm{EAC}$ group for clarithromycin were $\mathrm{MIC}_{50},<0.016$ and $<0.016$ $\mathrm{mg} / \mathrm{L}, \mathrm{MIC}_{90}, 0.032$ and $0.064 \mathrm{mg} / \mathrm{L}$, and ranges of <0.016- $>256$ and <0.016-48 mg/L, respectively.

Successful eradication rates, as determined by a negative UBT after treatment, were similar in the patients of EAC group (80.6\%, 25 of 31) and in those of EACL group (83.9\%, 26 of $31, \mathrm{p}=0.740$, OR 0.80 ; 95\% CI: $0.22-2.96$ ) by both intention to treat and per protocol analysis. The strains of the 11 non-eradicated patients were in vitro susceptible to both clarithromycin and amoxicillin. The MIC ranges against the five strains of EACL group and the six strains of EAC group were $<0.016-0.016$ and $<0.016-0.19 \mathrm{mg} / \mathrm{L}$ for clarithromycin, and $<0.016-0.047 \mathrm{mg} / \mathrm{L}$ and $<0.016-0.19$ for amoxicillin, respectively.

Safety. There were no reported dropouts from the study due to non-compliance of the patients or side effects from the medications.

\section{DISCUSSION:}

Probiotics are live, nonpathogenic microbial feeds or food supplements that have been used to improve the balance of the microflora, primarily in the gastrointestinal tract [14]. Lactobacilli are acid resistant and can persist in the stomach longer than most other bacteria [15]; therefore, they have the potential to inhibit $H$. pylori, both for prophylaxis and for treatment of the infection.

Previous studies have found that the supplementation of standard anti- $H$. pylori regimens with probiotics can reduce the side effects of triple therapy and, therefore, can improve patients' compliance to therapy [14]. 
Other authors have identified the in vitro activity of L. acidophilus against H. pylori, which has been associated with the secretion of lactic acid, CRL639 autolysins and competition for adhesion [16]. However, most of these properties have been observed only in animal models or in vitro data. In addition, the activity of several probiotic species against $H$. pylori has been reported to be strain-dependant [17].

In vivo, the administration of probiotics alone usually does not lead to $H$. pylori eradication [16]. However, in several studies, adding Lactobacillus species (e.g., L. johnsonii La1 lyophilized + inactivated culture or L. casei DN 114001) to a triple therapy of amoxicillin, clarithromycin and a proton pump inhibitor has increased the eradication rate compared to the standard triple regimen alone [14].

In the present study, the addition of $1.5 \times 10^{10}$ L. acidophilus organisms in the morning and $1 \times 10^{10}$ organisms at night, failed to improve $H$. pylori eradication. Although ${ }^{13} \mathrm{C}$ UBT was performed at least six weeks after the end of therapy, the annual recurrence of $H$. pylori infection has been reported to be low $(<1-2.7 \%)$ in most developed countries [18]; therefore, UBT-positivity in our sample is most likely due to persistent infection than to reinfection, the latter being unlikely to influence the study outcome.

There are several possible reasons for the absence of effect observed in this study. Firstly, the choice of probiotic to be added to the standard therapy seems to be important. It is still unclear which Lactobacillus species and strains are most active against $H$. pylori. So far, the most effective (both in vitro and in vivo) Lactobacillus species have been L. casei and $L$. johnsonii La1 [16]. In the relatively few studies on the addition of L. acidophilus to the standard eradication regimens, only two sets of data have reported an increase in $H$. pylori eradication rate. However, in one of the successful studies, a probiotic preparation containing both L. acidophilus and L. rhamnosus had been used [5,7].

In addition, the length (14 days in the study by De Francesco et al. [6], three weeks in the study by Cremonini et al. [19] and 8 days in the present study) and frequency of administration (t.i.d. in the study of Canducci et al. ${ }^{5}$ and b.i.d. in the present study) could also be of importance.

Finally, a combination of probiotics could provide a better effect on $H$. pylori eradication than the use of single species. Several authors $[7,20]$ have obtained an increase in H. pylori eradication, compared to the standard therapy with probiotic combinations such as Lactobacillus- and Bifidobacterium-containing yogurt (AB-Yogurt) or L. acidophilus and L. rhamnosus. A recent study has demonstrated a significantly higher $H$. pylori eradication rate by a quadruple therapy following pre-treatment with $\mathrm{AB}$-yogurt containing a mixture of $L$. 
acidophilus La5, Bifidobacterium lactis Bb12, Lactobacillus bulgaricus, and Streptococcus thermophilus, than by the quadruple therapy alone [21].

\section{CONCLUSION}

$H$. pylori eradication rates in the EAC and EACL groups were comparable: adding $L$. acidophilus to the triple therapy used in this study did not increase the eradication rate. Considering both the patients' expenses and the need for the daily ingestion of five extra capsules, the supplementation of the amoxicillin + clarithromycin + esomeprazole therapy with L. acidophilus, at a daily dose of $2.5 \times 10^{10}$ cells, does not benefit the ulcer patient.

Further studies with different posologies or duration of treatment, and with alternative probiotics or probiotic combinations are required to improve the eradication of $\mathrm{H}$. pylori infection.

Conflict of interest statement: The authors declare that they have no conflicts of interest, including financial or personal relationships with other people or organisations, that could influence their work.

\section{ACKNOWLEDGMENTS}

The authors would like to thank the "Dr. Ricardo Jorge" National Institute of Health, Portugal, for its support in the execution of the microbiology tests. 


\section{References}

1. Megraud F (2004) H pylori antibiotic resistance: prevalence, importance, and advances in testing. Gut 53:1374-84. doi: 10.1136/gut.2003.022111

2. Hamilton-Miller JM (2003) The role of probiotics in the treatment and prevention of Helicobacter pylori infection. Int J Antimicrob Agents 22:360-366. doi:10.1016/S09248579(03)00153-5

3. Sgouras D, Maragkoudakis P, Petraki K, Martinez-Gonzalez B, Eriotou E, Michopoulos S, Kalantzopoulos G, Tsakalidou E, Mentis A (2004) In vitro and in vivo inhibition of Helicobacter pylori by Lactobacillus casei strain Shirota. Appl Environ Microbiol 70:518-526. doi:10.1128/AEM.70.1.518-526.2004

4. Ushiyama A, Tanaka K, Aiba Y, Shiba T, Takagi A, Mine T, Koga Y (2003) Lactobacillus gasseri OLL2716 as a probiotic in clarithromycin-resistant Helicobacter pylori infection. J Gastroenterol Hepatol 18:986-991. doi:10.1046/j.14401746.2003.03102.x

5. Canducci F, Armuzzi A, Cremonini F, Cammarota G, Bartolozzi F, Pola P, Gasbarrini G, Gasbarrini A (2000) A lyophilized and inactivated culture of Lactobacillus acidophilus increases Helicobacter pylori eradication rates. Aliment Pharmacol Ther 14:1625-1629. doi:10.1046/j.1365-2036.2000.00885.x

6. De Francesco V, Stoppino V, Sgarro C, Faleo D (2000) Lactobacillus acidophilus administration added to omeprazole/amoxycillin-based double therapy in Helicobacter pylori eradication. Dig Liver Dis 32:746-747. doi:10.1016/S1590-8658(00)80343-6

7. Ziemniak W (2006) Efficacy of Helicobacter pylori eradication taking into account its resistance to antibiotics. J Physiol Pharmacol 57 (Suppl 3):123-141.

8. Canducci F, Cremonini F, Armuzzi A, Di Caro S, Gabrielli M, Santarelli L, Nista E, Lupascu A, De Martini D, Gasbarrini A (2002) Probiotics and Helicobacter pylori eradication. Dig Liver Dis 34 (Suppl 2):S81-S83. doi:10.1016/S1590-8658(02)80172-4

9. Sakamoto I, Igarashi M, Kimura K, Takagi A, Miwa T, Koga Y (2001) Suppressive effect of Lactobacillus gasseri OLL 2716 (LG21) on Helicobacter pylori infection in humans. J Antimicrob Chemother 47:709-710. doi:10.1093/jac/47.5.709

10. Van Leerdam ME, van der Ende A, ten Kate FJ, Rauws EA, Tytgat GN (2003) Lack of accuracy of the noninvasive Helicobacter pylori stool antigen test in patients with gastroduodenal ulcer bleeding. Am J Gastroenterol 98:798-801. 
11. Monteiro L, Cabrita J, Mégraud F (1997) Evaluation of performances of three DNA enzyme immunoassays for detection of Helicobacter pylori PCR products from biopsy specimens. J Clin Microbiol 35:2931-2936.

12. Megraud F, Lehn N, Lind T, Bayerdörffer E, O'Morain C, Spiller R, Unge P, van Zanten SV, Wrangstadh M, Burman CF (1999) Antimicrobial susceptibility testing of Helicobacter pylori in a large multicenter trial: the MACH 2 study. Antimicrob Agents Chemother 43:2747-2752.

13. Huibers MJ, Bleijenberg G, Beurskens AJ, Kant IJ, Knottnerus JA, van der Windt DA, Bazelmans E, van Schayck CP (2004) An alternative trial design to overcome validity and recruitment problems in primary care research. Fam Pract 21:213-218. doi:10.1093/fampra/cmh219

14. Franceschi F, Cazzato A, Nista EC, Scarpellini E, Roccarina D, Gigante G, Gasbarrini G, Gasbarrini A (2007) Role of probiotics in patients with Helicobacter pylori infection. Helicobacter 12 (Suppl 2):59-63. doi:10.1111/j.1523-5378.2007.00565.x

15. Bhatia SJ, Kochar N, Abraham P, Nair NG, Mehta AP (1989) Lactobacillus acidophilus inhibits growth of Campylobacter pylori in vitro. J Clin Microbiol 27: 2328-30.

16. Lesbros-Pantoflickova D, Corthésy-Theulaz I, Blum AL (2007) Helicobacter pylori and probiotics. J Nutr 137(3 Suppl 2):812S-818S.

17. Kim TS, Hur JW, Yu MA, Cheigh CI, Kim KN, Hwang JK, Pyun YR. Antagonism of Helicobacter pylori by bacteriocins of lactic acid bacteria. J Food Prot 2003;66:3-12.

18. Niv Y (2008) H pylori recurrence after successful eradication. World J Gastroenterol 14, 1477-1478. doi: 10.3748/wjg.14.1477.

19. Cremonini F, Di Caro S, Covino M, Armuzzi A, Gabrielli M, Santarelli L, Nista E, Cammarota G, Gasbarrini G, Gasbarrini A (2002) Effect of different probiotic preparations on anti-Helicobacter pylori therapy-related side effects: a parallel group, triple blind, placebo-controlled study. Am J Gastroenterol 97:2744-2749. doi:10.1111/j.1572-0241.2002.07063.x

20. Sheu B, Wu J, Lo C, Wu H, Chen J, Lin Y, Lin M (2002) Impact of supplement with Lactobacillus- and Bifidobacterium-containing yogurt on triple therapy for Helicobacter pylori eradication. Aliment Pharmacol Ther 16:1669-1675. doi:10.1046/j.13652036.2002.01335.x

21. Sheu BS, Cheng HC, Kao AW, Wang ST, Yang YJ, Yang HB, Wu JJ (2006) Pretreatment with Lactobacillus- and Bifidobacterium-containing yogurt can improve the efficacy of 
quadruple therapy in eradicating residual Helicobacter pylori infection after failed triple therapy. Am J Clin Nutr 83:864-869.

Table 1. Patients involved in the study.

\begin{tabular}{|c|c|c|c|}
\hline Group & Parameter & $\begin{array}{l}\text { Patients in the EACL } \\
\text { group }\end{array}$ & $\begin{array}{l}\text { Patients in the } \\
\text { EAC group }\end{array}$ \\
\hline \multirow[t]{2}{*}{ Sex } & Men & $18(58.1 \%)$ & $14(45.2 \%)$ \\
\hline & Women & $13(41.9 \%)$ & $17(54.8 \%)$ \\
\hline \multirow[t]{3}{*}{ Age } & Average (years) & 50.7 & 53.8 \\
\hline & Median (years) & 52.0 & 58.5 \\
\hline & Range (years) & $22-80$ & $24-78$ \\
\hline \multirow[t]{3}{*}{ Diseases } & Duodenal ulcer & $20(64.5 \%)$ & $23(74.2 \%)$ \\
\hline & Duodenal ulcer scar & $10(32.3 \%)$ & $5(16.1 \%)$ \\
\hline & Gastric ulcer & $1(3.2 \%)$ & $3(9.7)$ \\
\hline Total & All patients & 31 & 31 \\
\hline
\end{tabular}

Abbreviations: EAC group: patients treated by the standard triple therapy - esomeprazole 20 mg, amoxicillin $1000 \mathrm{mg}$ and clarithromycin $500 \mathrm{mg}$, all b.i.d. for 8 days; EACL group: patients treated by the triple therapy plus L. acidophilus $-5 \times 10^{9}$ organisms per capsule, 3 capsules in the morning and 2 capsules at night, for the same period of time. 
Table 2. H. pylori eradication by adding Lactobacillus acidophilus to the eradication regimens of patients with gastroduodenal diseases.

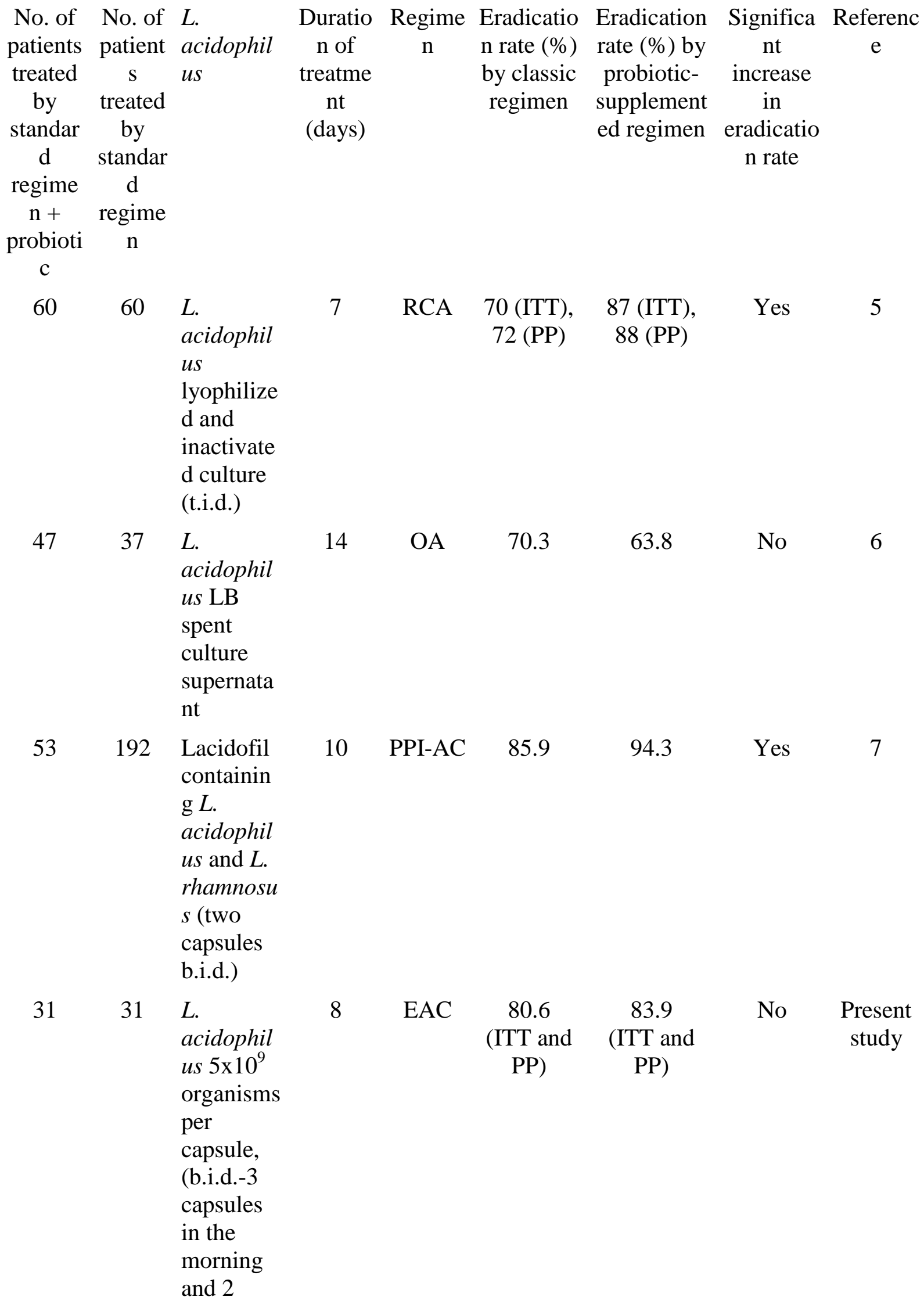


capsules

at nigh)

Abbreviations: ITT: intention to treat analysis., PP: per protocol analysis; RCA: rabeprazole (20 mg b.i.d.), clarithromycin (250 mg t.d.s.) and amoxicillin (500 mg t.d.s.); OA: omeprazole + amoxicillin; EAC: esomeprazole 20 mg b.i.d., amoxicillin $1000 \mathrm{mg}$ b.i.d. and clarithromycin $500 \mathrm{mg}$ b.i.d., for 8 days; PPI-AC: amoxicillin with clarithromycin and proton pump inhibitor. 
Table 1. Patients involved in the study.

\begin{tabular}{|c|c|c|c|}
\hline Group & Parameter & $\begin{array}{l}\text { Patients in the EACL } \\
\text { group }\end{array}$ & $\begin{array}{l}\text { Patients in the } \\
\text { EAC group }\end{array}$ \\
\hline \multirow[t]{2}{*}{ Sex } & Men & $18(58.1 \%)$ & $14(45.2 \%)$ \\
\hline & Women & $13(41.9 \%)$ & $17(54.8 \%)$ \\
\hline \multirow[t]{3}{*}{ Age } & Average (years) & 50.7 & 53.8 \\
\hline & Median (years) & 52.0 & 58.5 \\
\hline & Range (years) & $22-80$ & $24-78$ \\
\hline \multirow[t]{3}{*}{ Diseases } & Duodenal ulcer & $20(64.5 \%)$ & $23(74.2 \%)$ \\
\hline & Duodenal ulcer scar & $10(32.3 \%)$ & $5(16.1 \%)$ \\
\hline & Gastric ulcer & $1(3.2 \%)$ & $3(9.7)$ \\
\hline Total & All patients & 31 & 31 \\
\hline
\end{tabular}

Abbreviations: EAC group: patients treated by the standard triple therapy - esomeprazole 20 $\mathrm{mg}$, amoxicillin $1000 \mathrm{mg}$ and clarithromycin $500 \mathrm{mg}$, all b.i.d. for 8 days; EACL group: patients treated by the triple therapy plus L. acidophilus $-5 \times 10^{9}$ organisms per capsule, 3 capsules in the morning and 2 capsules at night, for the same period of time. 
Table 2. H. pylori eradication by adding Lactobacillus acidophilus to the eradication regimens of patients with gastroduodenal diseases.

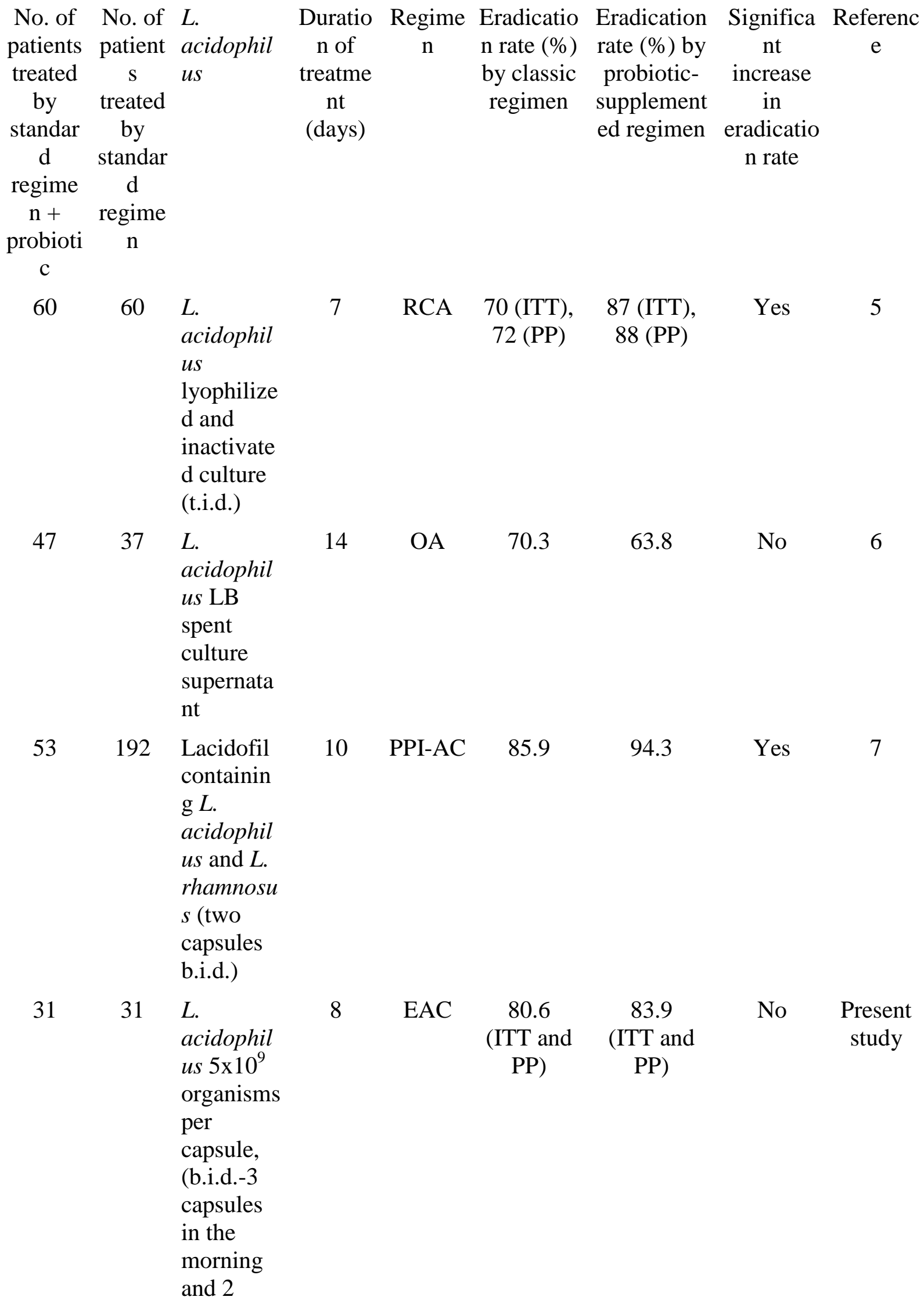


capsules

at nigh)

Abbreviations: ITT: intention to treat analysis., PP: per protocol analysis; RCA: rabeprazole (20 mg b.i.d.), clarithromycin (250 mg t.d.s.) and amoxicillin (500 mg t.d.s.); OA: omeprazole + amoxicillin; EAC: esomeprazole 20 mg b.i.d., amoxicillin $1000 \mathrm{mg}$ b.i.d. and clarithromycin $500 \mathrm{mg}$ b.i.d., for 8 days; PPI-AC: amoxicillin with clarithromycin and proton pump inhibitor. 